\title{
National protocol for quality assurance in DXA-bone densitometry
}

\author{
National Centre of Radiobiology and Radiation Protection, Sofia, Bulgaria \\ e-mail: a.slavchev@ncrrp.org
}

\begin{abstract}
Osteoporosis becomes largely one of the most important socially significant and costly diseases. Modern techniques (DXA, US) are applied for bone densitometry. The paper presents a protocol for quality assurance especially of DXA-bone densitometers including quality control made in compliance with international standards (ISCD, IOF). The methodology has been tested in practice by measurements on site-functional assessment, entrance dose, radiation protection, calibration, in-vitro precision. It is expected to raise the quality of the diagnostic process in concert with the $\mathrm{EU}$ Medical Directive 97/43 particularly for population screening and sensitive groups. The protocol is an essential part of the National Program for constraining osteoporosis which has been elaborated at the Ministry of Health and at present under implementation throughout the country. It aims at reducing the risk, factors spreading, at diminishing the fracture risk the morbidity and the mortality from osteoporosis. An integral multidisciplinary approach to the problem solving is applied as well as training on three levels - doctors, patients, population, which effectively will contribute for obtaining real results in preventing osteoporosis.
\end{abstract}

Key words: DXA bone densitometry, quality assurance protocol, QC measurements.

\section{Introduction}

The European Medical Exposure Directive EURATOM 97/43 [3] and the corresponding national regulations lay down severe requirements onto the quality assurance (QA) and the regular quality control (QC) of the radiological equipment including calibration and assessment of safety, accuracy and precision among others. The accomplishment of tests (initial, periodic, on request, etc.) is obligatory in order to guarantee a proper and 
reliable operation of the concerned equipment, under strict adhering to the manufacturer instructions. When new machines have to be put into operation or novel techniques (new applications) have to be checked before introducing into the clinical practice, certain measurements have to be done additionally. The same applies when for example after significant service procedures or serious repairs the working conditions changed and could cause possible deteriorations. The QA program includes the regular and continuing training of the operating staff and keeping the relevant documentation in order.

Dual Energy X-Ray Absorptiometry (DXA) is a well established, clinically proven 'gold standard' method for bone densitometry especially with view to the diagnostics and treatment of osteoporosis. In our days, already the fourth generation of DXA densitometers (fan and cone beam, digital) is advancing and the older machines (pencil beam) still are working successfully - a great variety of models. When adhering to the respective manufacturer prescriptions for operation and service the warranty is given that the machine parameters will remain stable in the nominal range and the highest degree of accuracy and precision will be achieved. Further, the radiation risk for the patients and the staff will be kept at minimum. This is particularly important because of the increased patient dose with the latest DXA models and the higher patient throughput. This fact necessarily leads to additional scattered radiation and occupational risk. Thus, a regular QC including radiation protection and performance checks is mandatory.

Based on the EC Regulations and depending on the national rules different types of QC protocols are elaborated and practiced in the different countries. In order to standardize and to make results internationally compatible the issued guidances of the International Society on Clinical Densitometry (ISCD) are published in the form of regular Official positions [5]. In the scope of the FP6 SENTINEL project a study of the DXA densitometric practice in some EU countries has been performed by means of a unified protocol [4].

\section{Material and methods}

On the basis of the unified protocol and further recommendations a Methodic Instruction for QC of DXA densitometers [4] has been drafted and tested in practice by measurements on site following the items of the corresponding protocol. The practical 
implementation was well favored by the realization of the National Program for preventing osteoporosis initiated by the Ministry of Health and at present already in realization throughout the country. As usual the players in the National Program are the Osteoporosis Centres (bone densitometry clinical centers), the regional centres (University hospital, professional body), indirectly the manufacturers of densitometry equipment and independent organizations (control and audit).

A guiding number of DXA densitometers for an optimal management of osteoporosis has been recommended [6] as 10-11 per million population. It then follows that about at least 70 machines would be necessary for a country like Bulgaria. Here, of the 56 Centres for osteoporosis 20 possess DXA densitometers (whole body, peripheral and others). During the first phase the protocol has been tested on 8 DXA machines of the $3 \mathrm{~d}$ generation-pencil beam. Some of them were new ones, others - second hand imported. Most of the machines undergo regular service checks. All densitometers perform the specific daily calibration checks, prescribed by the manufacturer who guarantees for stable and proper operation, i.e., a high degree of accuracy.

Since the most important characteristics of the densitometers are accuracy and precision the protocol itself has been divided in three parts: functional assessment, radiation protection and patient safety and in-vitro precision. It entirely corresponds to that exercised in the SENTINEL study: equipment survey, QC, patient dose, bone mineral density (BMD) accuracy and precision. The Methodic instruction presents a detailed description of how to perform the measurements and how to fill in the protocol elaborated in-house [7].

In the first part of the protocol basic technical information on the DXA system and the radiation protection of the facility is input: $\mathrm{kVp}, \mathrm{mA}$, scanning mechanism, tube position, detector type, warning lights and signs, shutter, ON/OFF switches, emergency, regular QC, individual competencies of the operator.

Targets of the quality control are the physical-technical parameters and their permissible variations (remedial and suspension limits). The $\mathrm{QC}$ measurements require dosemeter with two ionization chambers - one for radiation protection measurements with a volume $1000-1800 \mathrm{~cm}^{3}$ and another ionization chamber with volume $70-180 \mathrm{~cm}^{3}$ for patient dose and half value layer measurements. For the QC further are needed: calibration and/ or anthropomorphic phantoms, a water equivalent (water or PMMA) phantom with $20 \mathrm{~cm}$ thickness, a set of aluminum filters, a film cassette or CR plate. Parameters to be preferably measured are: radiation field size, tube output 
consistency (repeatability), Half Value Layer (HVL), patient entrance surface air kerma (ESAK), kerma-area product (KAP) and scattered radiation.

The radiation field size was measured by placing a film cassette on the table top and scanning in standard lumbar spine mode. The film was developed and the field size measured.

The consistency of the tube output was estimated by placing the small ionization chamber onto the table and performing three scans in the lumbar spine mode for a standard patient. The coefficient of variation of the results was calculated.

HVL was measured with the small chamber positioned $10 \mathrm{~cm}$ above the table top and by placing $\mathrm{Al}$ filters of different thickness underneath (in all checked densitometers the X-ray tube was under couch). For each $\mathrm{Al}$ filter the air kerma was measured during scanning in the lumbar spine mode for a standard patient. The measurements have been repeated and the HVL was determined in the usual way.

Patient entrance surface air kerma was measured on the entrance surface of the water phantom, positioned about $3 \mathrm{~cm}$ above the table, the small ionization chamber lying on the table for different scan modes (standard, thin, thick patient). KAP was then calculated from the measured values and the corresponding area. The calculated values may be used for comparative studies between different DXA machines.

Scattered radiation was measured at different distances from the phantom (water or PMMA), placed on the table top with the big ionization chamber, vertically and horizontally aligned with the phantom. The measurements have been executed for the different scan modes.

In all control measurements the scan parameters - $\mathrm{mA}$, time and focus-chamber or focus-film distance have always been recorded.

The machine performance (accuracy) was checked by repeating the daily calibration checks and the QC measurements with the help of a producer specific phantom in lumbar spine mode.

The in-vitro precision measurements by means of the anthropomorphic phantom were focused on the operator's abilities and other subjective factors for an adequate and reliable diagnostics. As to the ISCD recommendations the short-term in-vitro precision has been tested by 10 successive scans of the anthropomorphic phantom with repositioning in standard patient mode. This phantom is of known BMD although in different form and structure from the diverse manufacturers - for example: an Al slab simulating L1-L4 vertebrae in a $15 \mathrm{~cm}$ water bath, a simulated spine embedded in epoxy 
block or similar model in a cube with acrylic filling. All the operators had an experience in measuring BMD of more than 500 patients.

After the measurements have been done on the correspondent machine the standard deviation (SD) $\left[\mathrm{g} / \mathrm{cm}^{2}\right]$ and the coefficient of variation (CV) [\%] of the measured $\mathrm{BMD}$ (BMC) have been calculated and the respective precision $\mathrm{Pr}=\mathrm{CV}[\%]$ derived. The $\mathrm{SD}$ and the $\mathrm{CV}$ are calculated as follows:

$$
\mathrm{SD}=\sqrt{\frac{\sum_{i=1}^{n}\left(X_{i}-\bar{X}\right)^{2}}{n-1}}, \quad \mathrm{CV}=\frac{\mathrm{SD}}{\bar{X}} \cdot 100
$$

where $\bar{X}\left[\mathrm{~g} / \mathrm{cm}^{2}\right]$ is the arithmetic mean of the BMD (BMC) measurements, $n-$ the number of measurements, and $X_{i}$ - the result from the $i$-measurement. This has been referred as a preliminary base for the short-term in-vitro precision.

The space resolution tests which are of growing importance for the new diagnostic methods - VFA, AAC, whole body composition analysis, have been executed by means of Huttner test objects. Other parameters are of interest as well, namely linearity, image quality and others.

\section{Results and discussion}

\section{Radiation protection survey}

It showed a good organization and disposition of the concerned premises. All of them are designed and equipped as to the legal requirements for work with sources of ionizing radiation. In most cases care is taken that no person is unnecessarily exposed to radiation. In one case certain measures have to be undertaken to ensure good air conditioning since the densitometer turned out to be very temperature sensitive.

\section{Radiation measurements}

The results from the measurements in 8 densitometry centres are given in Table 1 .

a) Radiation field size: The remedial limits in the protocol require difference between the nominal and the measured irradiated field size no more than $1 \mathrm{~cm}$ - row 1 in Table 1. One of the densitometers is constructed in such a way that it restricts the 
Table 1. The results from the measurements in eight densitometry centres

\begin{tabular}{|c|c|c|c|c|c|c|c|c|}
\hline BD Centre & 1 & 2 & 3 & 4 & 5 & 6 & 7 & 8 \\
\hline $\begin{array}{l}\text { Diff. nominal/measured irradiated } \\
\text { field size (length/ width) }[\mathrm{cm}]\end{array}$ & $0.3 / I$ & $\begin{array}{l}0.3 / \\
0.1\end{array}$ & $\begin{array}{l}0.2 / \\
0.7\end{array}$ & $\begin{array}{c}0 / \\
-1.5\end{array}$ & $\begin{array}{c}0 / \\
0.95\end{array}$ & $\begin{array}{l}0.05 / \\
0.95\end{array}$ & $\begin{array}{l}0.15 / \\
1.15\end{array}$ & $\begin{array}{l}3.0 / \\
2.7\end{array}$ \\
\hline $\begin{array}{l}\mathrm{CV} \text { of a series of } \\
\text { three measurements [\%] }\end{array}$ & n.a. & n.a. & 0.87 & 0.27 & 1.12 & 0.42 & 1.24 & 6.83 \\
\hline HVL [mmAl] & 4.0 & 3.3 & 3.6 & 4.3 & 3.4 & 3.4 & 6.0 & 6.0 \\
\hline $\operatorname{ESAK}[\mu \mathrm{G} y]$ & 5.85 & 8.45 & 7.48 & 16.7 & 39.5 & 24.45 & 17.42 & 10.71 \\
\hline $\mathrm{KAP}\left[\mathrm{mGy} \cdot \mathrm{cm}^{2}\right]$ & 2.11 & 3.04 & 2.27 & 5.59 & 9.77 & 4.64 & 3.31 & 2.89 \\
\hline $\begin{array}{l}\text { Scattered radiation at } 1 \mathrm{~m} \\
\text { distance }[\mu \mathrm{S} v / \mathrm{h}]\end{array}$ & 0.6 & 1.0 & 0.01 & 0.4 & 0 & 0 & 0.2 & 0.95 \\
\hline
\end{tabular}

field size depending on the object scanned. The symbol '/ stands for it because the width of the field size is decreased during the scanning process. In two cases deviations $(<1.5 \mathrm{~cm})$ from the set field size have been found.

b) Radiation output consistency: The measurements to check the tube output consistency were realized by three successive scans in the lumbar spine mode for a standard patient. As it may be seen the machines exhibit a good reproducibility of the generated output in all cases except one (row 2 in Table 1). The remedial limit in the protocol for this parameter is $5 \%$. The extremely high variation in the last case was caused by temperature fluctuations. When the room became well conditioned the repeated measurements showed a coefficient of variation below the limits.

c) Half-value-layer: The measured values of HVL were set as a baseline during the commissioning measurements. The values which will be measured during the periodic tests should be compared with the baseline. It could be realized (row 3 in Table 1) that in different systems certain variations in HVL are present.

d) Patient dose: The patient dose is dependent on the source-detector distance, the source collimation, the tube current and the energy spectra. Reducing the patient 
dose in most cases leads to reduction of the staff dose. As is witnessed (rows 4 and 5 in Table 1) the patient doses are in line with or even lower than those values specified by the manufacturers: $20-50 \mu \mathrm{Gy}$. An additional optimization in terms of a further reduction of the patient dose could be achieved by means of the radiation protection choice of appropriate scan protocol, well skilled positioning, etc. It becomes exceptionally important for the fan and cone beam densitometers where the doses are higher. The dose variations for the different scan protocols should be investigated as well.

e) Scatter radiation: Measurements of scattered radiation have been made at least three locations around the patient table, including the operator place. Scattered radiation dose rates measured at $1 \mathrm{~m}$ at the different DXA machines (after subtracting the background) in order to compare with the specified value by the manufacturers (about $1 \mu \mathrm{Sv} / \mathrm{h}$ at a distance of $1 \mathrm{~m}$ from the scanning head) are presented in row 6 of Table 1. The low dose rate levels were expected for the pencil beam systems. The radiation dose to the operator depends on the densitometer itself, the patient throughput, the distance from the table, available radiation protective tools and others. As it may be seen from Table 1 the annual scatter dose for a typical workload $(1300 \mathrm{~h})$ at $1 \mathrm{~m}$ distance with the maximum of $1 \mu \mathrm{Sv} / \mathrm{h}$ would be between $13 \mu \mathrm{S} v$ and $1300 \mu \mathrm{S} v$ or $0.013 \mathrm{mSv}$ per year and $1.3 \mathrm{mS} v$ per year at maximum.

In all five tests (a-e) good compliance with the reported SENTINEL results is evident [4].

\section{Accuracy}

The accuracy has been tested by 5-10 measurements of the QC phantom without repositioning. In all cases the obtained BMD values were within the tolerances specified by the producer $(0.5-1.0 \%)$. In two cases (one with the temperature fluctuations and the other with some service shortage) the difference was slightly greater than $1.0 \%$. In the first case after the room conditioning the repeatability of the measurements was excellent. The second one needed some service. The present study illustrated how important are the regular quality control of the densitometer and a consistent calibration procedure. The long term monitoring of the machine parameter stability and the regular performance checks are of viable significance for the adequacy and reliability of the BMD measurements. 


\section{Short-term precision (repeatability) in-vitro}

The calculated precision then was compared to that specified by the manufacturer Table 2.

In one case (column 7) measurements have been made with the phantom upside down to check precision for specific patient group. The slight difference does not impact radically the values. For a more general conclusion further measurements are needed. At this point the fundamental ISCD recommendation has to be accounted for that the

Table 2. Comparing the calculated precision and specified one by the manufacturer

\begin{tabular}{|c|c|c|c|c|c|c|c|c|}
\hline BD Centre & 1 & 2 & 3 & 4 & 5 & 6 & 7 & 8 \\
\hline \hline SD [g.cm & -2 \\
\hline CV [\%] $=$ Prec & 0.0039 & 0.0034 & 0.004 & 0.004 & 0.007 & 0.011 & $\begin{array}{c}0.019 / \\
0.017\end{array}$ & 0.027 \\
\hline
\end{tabular}

producer specified in-vitro precision values shouldn't be used for the clinical practice rather the range tolerances. It is due to the BMD non-consistency between manufacturers, among others. The ISCD recommended minimum precision for the lumbar spine is $\mathrm{CV}=1.9 \%$. As is well evident only one case was very unlike. It namely was that one due to temperature fluctuations. After the room became well-conditioned, precision showed nominal value alike. In the next stage the short-term and the long-term precision in-vivo of each densitometric centre should be assessed.

\section{Conclusions}

The experience from different models of DXA densitometers operating at different sites throughout the country turned out that at present the machines perform in compliance with the referral guidelines set by the international organizations (ISCD, ICRP, EC Directives) and the manufacturers. The study illustrated a comprehensive accuracy and a comparable densitometric practice with that exercised within the EU. Thus, results may be internationally acknowledged and used for comparative analysis or research 
trials. In certain cases corrective actions were necessary with regard to environmental conditions and the operator training in radiation protection.

The QC tests are important for the frequency of the follow up scanning alike, and should continue and be extended by comparative trials to stay in concordance with the international trends and to share advanced professional experience. The steady dose monitoring is essential for establishing diagnostic reference levels and later on for the elaboration of a medical standard for bone densitometry. It is henceforth of particular significance for the successful application of the National Program for Constraining Osteoporosis especially when its screening part is started.

\section{References}

[1] Accreditation Guidelines for Bone Densitometry. Australian and New Zealand Bone and Mineral Society; June 2004.

[2] Cawte SA, Pearson D et al. Cross-calibration, Precision and Patient Dose Measurements in Preparation for Clinical Trials using DXA of Lumbar Spine. BJR. 1999; 72: 354-362.

[3] Council Directive 97/43/EURATOM of 30 June 1997 on health protection of individuals against the dangers of ionizing radiation in relation to medical exposure, and repealing Directive 84/466/Euratom. OJ L. 1997 Jul 9; 180: 22-27, 9.7.1997.

[4] DXA Radiation Protection and Quality Assurance. Sentinel Training Course. Dublin: October 2006.

[5] Baim S, Wilson CR, Lewiecki EM, Luckey MM, Downs RW, Lentle BC. Precision Assessment and Radiation Safety for Dual-Energy X-Ray Absorptiometry: Position Paper of the International Society for Clinical Densitometry. JCD. 2005; 8(4).

[6] Kanis JA, Johnell O. Requirements for DXA for the Management of Osteoporosis in Europe. Osteoporosis Int. 2006; 16: 229-238.

[7] Methodic Instruction for Quality Control of DXA Densitometers - Draft. NCRRP, MoH, Sofia, 2008.

[8] Radiological Safety of DXA Bone Densitometry Equipment. Radiation Issue Notes. BC Centre for Disease Control, July 2001. 